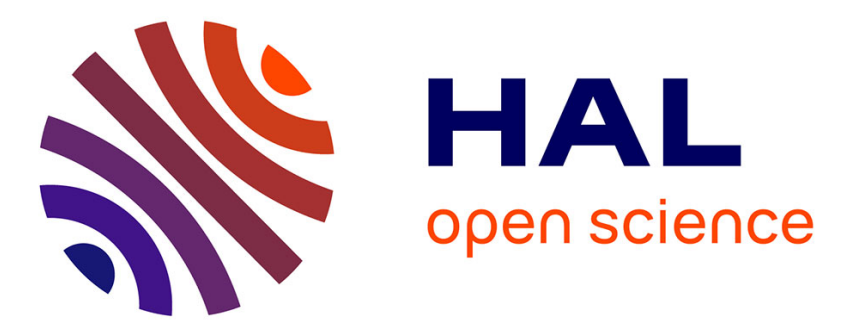

\title{
Impulsive Interference Mitigation in Ad Hoc Networks Based on Alpha-Stable Modeling and Particle Filtering
}

Nouha Jaoua, Emmanuel Duflos, Philippe Vanheeghe, Laurent Clavier, François Septier

\section{- To cite this version:}

Nouha Jaoua, Emmanuel Duflos, Philippe Vanheeghe, Laurent Clavier, François Septier. Impulsive Interference Mitigation in Ad Hoc Networks Based on Alpha-Stable Modeling and Particle Filtering. International Conference on Acoustics, Speech and Signal Processing (ICASSP), 2011, May 2011, Prague, Czech Republic. pp.3548 - 3551, 10.1109/ICASSP.2011.5946244 . hal-00640682

\section{HAL Id: hal-00640682}

\section{https://hal.inria.fr/hal-00640682}

Submitted on 14 Nov 2011

HAL is a multi-disciplinary open access archive for the deposit and dissemination of scientific research documents, whether they are published or not. The documents may come from teaching and research institutions in France or abroad, or from public or private research centers.
L'archive ouverte pluridisciplinaire HAL, est destinée au dépôt et à la diffusion de documents scientifiques de niveau recherche, publiés ou non, émanant des établissements d'enseignement et de recherche français ou étrangers, des laboratoires publics ou privés. 


\title{
IMPULSIVE INTERFERENCE MITIGATION IN AD HOC NETWORKS BASED ON ALPHA-STABLE MODELING AND PARTICLE FILTERING
}

\author{
Nouha Jaoua ${ }^{1}$,Emmanuel Duflos ${ }^{1}$, Philippe Vanheeghe ${ }^{1}$, Laurent Clavier ${ }^{2,3}$, François Septier ${ }^{3}$ \\ ${ }^{1}$ LAGIS FRE CNRS 3303, Ecole Centrale de Lille, 59651 Villeneuve d'Ascq, France. \\ ${ }^{2}$ IRCICA FR CNRS 3024, Université de Lille 1, 59650 Villeneuve d'Ascq, France. \\ ${ }^{3}$ Institut TELECOM, TELECOM Lille 1, 59658 Villeneuve d'Ascq, France.
}

\begin{abstract}
In this paper, we tackle the problem of interference mitigation in ad hoc networks. In such context, the multiple access interference (MAI) is known to be of an impulsive nature. Therefore, the conventional Gaussian assumption can not be considered to model this type of interference. Contrariwise, it can be accurately modeled by stable distributions. Here, this issue is addressed within an Orthogonal Frequency Division Multiplexing (OFDM) transmission link assuming a symmetric $\alpha$-stable model for the signal distortion due to MAI. For this purpose, we propose a method for the joint estimation of the transmitted multicarrier signal and the noise parameters. Based on sequential Monte Carlo (SMC) methods, the proposed scheme allows the online estimation using a Raoblackwellized particle filter.
\end{abstract}

Index Terms- ad hoc networks, multiple access interference, sequential Monte Carlo methods, $\alpha$-stable distribution

\section{INTRODUCTION}

Sensor networks need to reduce the energy to sense and transmit data. Signal processing has a key role to play in order to minimize the transmission power necessary to ensure the required link quality and reliability. Interference in low power communications could however be a strong limitation. In $a d$ hoc networks, capacities of relay and interference channels are not analytically known.

If recent works from Bresler et al. [1] have approximated such a capacity, the way to cope with interference is still an open question. The authors suggest some interference alignment scheme whereas Dabora et al. [2] propose to use relays to forward interference in order to properly remove it at the receiver and obtain an interference free link. However, in sensor networks, the hard constraints on consumption and cost make it difficult to implement those complex proposals.

We are then interested in exploiting the non Gaussian behavior of the interference in order to limit its effects. Indeed, the ad hoc configuration gives to the multiple access interference (MAI) an impulsive nature: the interfering pulses amplitude may importantly vary and the MAI is conditioned by the presence of strong interferers. Several works [3, 4] have proposed to use stable distributions to model it and a general mathematical framework was recently proposed by Win et al. in [5]. They show the theoretical validity of the stable approach for different situations in network interference, including ad hoc and cognitive radio. We also suggest that the ad hoc configuration gives the shape of the MAI distribution which is then accurately represented by symmetric $\alpha$-stable distributions [6].

In this paper, this modeling of interference is used in an OFDM transmission link and we try to reduce the signal distortion due to MAI. For this purpose, we propose a method for the joint estimation of the multicarrier signal and the noise parameters. The proposed scheme is based on Bayesian estimation using SMC methods. We propose a sequential approach although it is not necessarily justified in OFDM based transmission but we are here interested in the possibility to remove interference without adding delays in the signal processing and therefore in the transmission.

The paper is organized as follows: in section 2, we present some preliminary definitions regarding the $\alpha$-stable distribution and its properties. In section 3, we introduce the OFDM system's model and its state space representation. Section 4 is devoted to the description of the proposed particle filter (PF) to estimate both multicarrier signal and noise parameters. Simulation results are shown in section 5 and conclusions are drawn through section 6.

\section{STABLE DISTRIBUTIONS}

Stable distributions can be seen as a generalization of the Gaussian distribution in that sense that they are the only distributions to be stable in convolution, meaning that the sum of two $\alpha$-stable distributions is an $\alpha$-stable distribution. One difficulty is that they have no closed-form expressions for their probability density function (pdf) and cumulative distribution. They can be most conveniently described by their characteristic functions as follows [7]:

$$
\varphi(t)=\exp \left(i \mu t-\gamma|t|^{\alpha}[1+i \beta \operatorname{sign}(t) \omega(t, \alpha)]\right)
$$


where

$$
\omega(t, \alpha)=\left\{\begin{array}{lll}
\tan \frac{\alpha \pi}{2}, & \text { if } & \alpha \neq 1 \\
\frac{2}{\pi} \log |t|, & \text { if } & \alpha=1
\end{array}\right.
$$

Thus, the stable distribution is completely determined by four parameters $\alpha, \beta, \gamma$ and $\mu$ where:

- $\alpha \in[0,2]$ is the characteristic exponent. It measures the tails heaviness of distribution. A small value of $\alpha$ will imply a considerable probability mass in the tails of distribution, while a value of $\alpha$ close to 2 indicates more Gaussian type behavior.

- $\mu \in \mathbb{R}$ is the location parameter.

- $\gamma>0$ is the scale parameter. It determines the spread of density around the location parameter $\mu$.

- $\beta \in[-1,1]$ is the symmetry parameter. When $\beta=0$, the distribution is symmetric about $\mu$.

To denote an $\alpha$-stable distribution with parameters $\alpha, \beta, \gamma$ and $\mu$, we will use the following notation $\mathcal{S}_{\alpha}(\beta, \gamma, \mu)$. An $\alpha$-stable distribution is said to be standard when $\gamma=1$ and $\mu=0$. For standardized stable distribution we will use the notation $\mathcal{S}_{\alpha}(\beta)$.

$\alpha$-stable distribution pdf can be obtained analytically only for a few particular cases: $\alpha=2$, corresponding to the Gaussian distribution, $\alpha=1$ and $\beta=0$, yielding the Cauchy distribution, and finally $\alpha=\frac{1}{2}$ and $\beta=1$ for the Levy distribution.

\section{MODEL}

In this paper, we consider a dynamic state space model, widely used in communication, to represent OFDM systems [8].

The considered OFDM system is based on $N$ subcarriers. The transmitted signal, composed of $N$ samples $s_{t}$ with $t=0 \ldots N-1$, is generated through an inverse FFT. Before transmission, a cyclic prefix of length $N_{c p}$ is introduced in order to avoid intersymbol interference. The resulting signal is transmitted in a time varying frequency selective multipath channel with $L$ paths.

We define the transmitted multicarrier signal vector as:

$$
S_{t}=\left[\begin{array}{llll}
s_{t} & \cdots & s_{-L+1} & \mathbf{0}_{1 \times\left(N+N_{c p}-t-1\right)}
\end{array}\right]^{T}
$$

where $s_{k}=0$ if $k<0$.

The state equation of $S_{t}$ can be written in the matrix form as follows:

$$
S_{t}=A_{t} S_{t-1}+B_{t}
$$

where the transition matrix $A_{t}$ is defined as:

$$
\left[\begin{array}{cc}
\xi_{t}^{T} & \\
\mathbf{I}_{\left(N+N_{c p}+L-2\right)} & \mathbf{0}_{\left(N+N_{c p}+L-2\right) \times 1}
\end{array}\right]
$$

with

$\xi_{t}^{T}= \begin{cases}{\left[\begin{array}{ll}\mathbf{0}_{1 \times\left(N+N_{c p}+L-1\right)}\end{array}\right]^{T},} & \text { if } 0 \leq t \leq N-1 \\ {\left[\begin{array}{lll}\mathbf{0}_{1 \times(N-1)} & \mathbf{0}_{1 \times\left(N_{c p}+L-1\right)}\end{array}\right]^{T},} & \text { if } N \leq t \leq N+N_{c p}-1\end{cases}$

and $B_{t}$ is a circular Zero-mean Gaussian noise vector with the covariance matrix

$$
E\left[B_{t} B_{t}^{H}\right]=\left[\begin{array}{cccc}
\sigma_{b_{t}}^{2} & 0 & \cdots & 0 \\
0 & 0 & & \\
\vdots & & \ddots & \vdots \\
0 & \cdots & & 0
\end{array}\right]
$$

where

$$
\sigma_{b_{t}}^{2}= \begin{cases}1, & \text { if } 0 \leq t \leq N-1 \\ 0, & \text { if } N \leq t \leq N+N_{c p}-1\end{cases}
$$

The received multicarrier signal $r_{t}$ can be written as:

$$
r_{t}=H^{T} S_{t}+\rho_{t} u_{t}
$$

for $t=0 \ldots N+N_{c p}-1$, where $H$ is the channel impulsive response

$$
H=\left[\begin{array}{llll}
h_{0} & \cdots & h_{L-1} & 0_{1 \times\left(N+N_{c p}-1\right)}
\end{array}\right]^{T}
$$

Contrary to [8], we assume that $u_{t}$ is a symmetric $\alpha$-stable noise

$$
u_{t} \sim \mathcal{S}_{\alpha}(0)
$$

Exploiting the mixture of normal representation of a stable distribution, $u_{t}$ can be written as follows [9]:

$u_{t}=\sqrt{\lambda_{t}} w_{t} \quad$ where $\quad \lambda_{t} \sim \mathcal{S}_{\frac{\alpha}{2}}(1) \quad$ and $\quad w_{t} \sim \mathcal{N}(0,1)$

Therefore, the observation equation becomes

$$
r_{t}=H^{T} S_{t}+D_{t} w_{t} \quad \text { with } \quad D_{t}=\left[\rho_{t} \sqrt{\lambda_{t}}\right]
$$

\section{PARTICLE FILTER FOR INTERFERENCE MITIGATION}

In this paper, our main target is to jointly estimate the transmitted multicarrier signal vector $S_{t}$ and the parameters of the model $\lambda_{t}, \alpha$ and $\rho_{t}$. In the context of Bayesian estimation, we need to compute the joint posterior pdf $p\left(S_{t}, \lambda_{t}, \alpha, \rho_{t} \mid r_{0: t}\right)$. However, this pdf is analytically intractable. Therefore, we propose to use SMC methods in order to find an estimate of $p\left(S_{t}, \lambda_{t}, \alpha, \rho_{t} \mid r_{0: t}\right)$.

Following [10], we denote $\theta_{t}$ the time varying parameter vector given by:

$$
\theta_{t}=\left[\alpha, \phi_{\rho_{t}}, \lambda_{t}\right] \quad \text { where } \quad \phi_{\rho_{t}}=\ln \left(\rho_{t}^{2}\right)
$$

The joint posterior pdf can be written as follows:

$$
p\left(S_{t}, \theta_{t} \mid r_{0: t}\right)=p\left(S_{t} \mid \theta_{t}, r_{0: t}\right) p\left(\theta_{t} \mid r_{0: t}\right)
$$


In the previous expression, the first probability density can be computed analytically using the Kalman filter (KF). Since, conditionally on $\theta_{t}$, our model is linear and Gaussian. The probability $p\left(S_{t} \mid \theta_{t}, r_{0: t}\right)$ can be written as follows:

$$
p\left(S_{t} \mid \theta_{t}, r_{0: t}\right)=\mathcal{N}\left(m_{t \mid t}, P_{t \mid t}\right)
$$

where $m_{t \mid t}$ and $P_{t \mid t}$ are computed using a KF.

The second probability density is intractable and is approximated by a PF [11]:

$$
p\left(\theta_{t} \mid r_{0: t}\right) \approx \sum_{i=1}^{M} \omega_{t}^{(i)} \delta_{\theta_{t}^{(i)}}\left(\theta_{t}\right)
$$

where $\omega_{t}^{(i)}$ is the normalized weight associated to the ith particle.

Thus, we obtain the following estimate of the joint posterior pdf:

$$
p\left(S_{t}, \theta_{t} \mid r_{0: t}\right) \approx \sum_{i=1}^{M} p\left(S_{t} \mid \theta_{t}^{(i)}, r_{0: t}\right) \omega_{t}^{(i)} \delta_{\theta_{t}^{(i)}}\left(\theta_{t}\right)
$$

Like in [10], the importance function is then chosen as:

$$
p\left(\theta_{t} \mid \theta_{t-1}\right)=p\left(\alpha_{t} \mid \alpha_{t-1}\right) p\left(\phi_{\rho_{t}} \mid \phi_{\rho_{t-1}}\right) p\left(\lambda_{t}\right)
$$

where

$$
\begin{aligned}
& p\left(\phi_{\rho_{t}} \mid \phi_{\rho_{t-1}}\right) \sim \mathcal{N}\left(\phi_{\rho_{t-1}}, \delta_{\phi_{\rho}}^{2}\right) \quad p\left(\phi_{\rho_{0}}\right) \sim \mathcal{N}\left(0, \delta_{\phi_{\rho_{0}}}^{2}\right) \\
& p\left(\lambda_{t}\right) \sim \mathcal{S}_{\frac{\alpha}{2}}(1)
\end{aligned}
$$

Our PF has to deal with the estimation of the static parameter $\alpha$. However, this intensifies the degeneracy problem. Considering this lack, we adopt the approach of artificial parameter evolution described in $[11,10]$. Nevertheless, the main problem of this approach is the information loss. In [11], the authors propose a method, to remediate to this gap, based on sampling the updated values of the static parameter from a kernel smoothed density. Using this method helped to define the conditional density evolution of $\alpha$ as:

$$
p\left(\alpha_{t+1} \mid \alpha_{t}\right) \sim \mathcal{N}\left(\alpha_{t+1} \mid d \alpha_{t}+(1-d) \bar{\alpha}_{t}, h^{2} \sigma_{t}^{2}\right)
$$

with

$$
h^{2}=1-d^{2}=1-\left(\frac{3 \delta-1}{2 \delta}\right)^{2}
$$

where $\bar{\alpha}_{t}$ and $\sigma_{t}^{2}$ are respectively the mean and the variance matrix of the Monte Carlo approximation of $p\left(\alpha \mid r_{0: t}\right)$ and $\delta$ is a discount factor in $] 0,1]$.

Weights are then updated according to:

$$
\tilde{\omega}_{t}^{(i)} \propto \tilde{\omega}_{t-1}^{(i)} p\left(r_{t} \mid \theta_{t}^{(i)}, r_{t-1}\right)
$$

with

$$
p\left(r_{t} \mid \theta_{t}^{(i)}, r_{t-1}\right)=\mathcal{N}\left(r_{t}, H^{T} m_{t \mid t-1}^{(i)}, G_{t}^{(i)}\right)
$$

where $G_{t}^{(i)}$ is the innovation covariance matrix from the KF.

Finally, the proposed $\mathrm{PF}$ for interference mitigation is summarized in Algorithm 1:

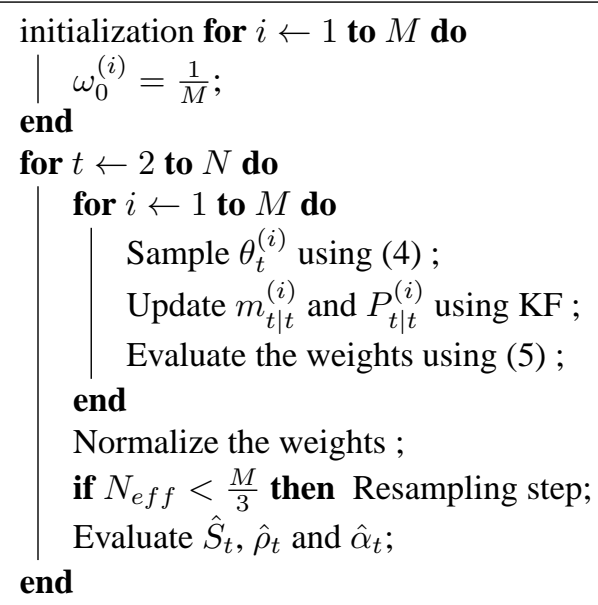

Normalize the weights ;

if $N_{\text {eff }}<\frac{M}{3}$ then Resampling step;

Evaluate $\hat{S}_{t}, \hat{\rho}_{t}$ and $\hat{\alpha}_{t}$;

end

Algorithm 1: PF for interference mitigation algorithm

\section{SIMULATIONS}

In order to validate our approach, various experiments have been carried out. We have considered the following system parameters: an OFDM system with $N=64$ subcarriers, a cyclic prefix of $N_{c p}=8$ samples and a multipath channel with $L=4$ paths. Both real and imaginary part of the transmitted multicarrier signal have been generated from a Gaussian distribution $\mathcal{N}\left(0, \frac{1}{2}\right)$ [8]. The proposed PF has been implemented with 100 particles. Parameters $\delta_{\phi_{\rho}}^{2}$ and $\delta_{\phi_{\rho_{0}}}^{2}$ are respectively set to 0.0005 and 0.5 . We fixed the discount factor $\delta$ to 0.95 [11].

At first, the algorithm performance is studied with a multicarrier signal corrupted by a symmetric $\alpha$-stable noise with $S N R=10$. The related parameters are set to: $\alpha=1.6$, $\mu=0$ and $\gamma=1$. Results are illustrated in the different plots of Fig. 1. The evolution of the estimated $\alpha$ and $\rho_{t}$ are respectively depicted in Fig. 1.(a) and Fig. 1.(b). We remark that, after some iterations, the estimated parameters start displaying the same trajectory as their actual counterparts. Curves in
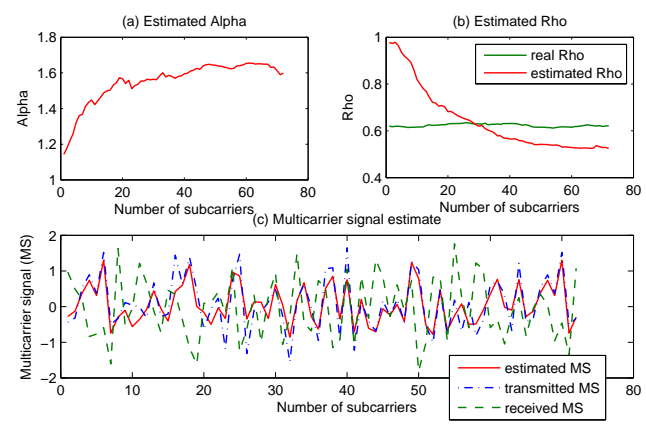

Fig. 1. The estimation of the model parameters and the multicarrier signal for $\alpha=1.6$ and $S N R=10$.

Fig. 1.(c) show the estimated multicarrier signal as well as the 
transmitted and the received ones. We can easily observe that the estimated multicarrier signal tends to be very close to the transmitted one.

Secondly, we study the performance of our method for different values of $\alpha$. For each one of them, we perform the experiment 100 times. The performances are shown in term of mean square error (MSE) of the multicarrier signal estimate. The average of the obtained MSEs are shown in Fig. 2.
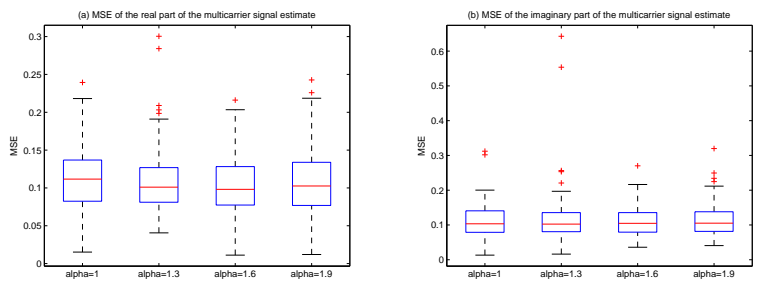

Fig. 2. Box and whiskers plots of the MSE of the multicarrier signal estimate for different values of $\alpha$.

In order to show the improvement brought by the $\alpha$-stable modelling, we have compared the previous estimation scheme to the following : the noise on the observations is $\alpha$-stable but the estimation process is carried out by supposing that the observation noise is simply gaussian. As it can be seen in Fig. 3, it is clear that including this impulsive nature in the estimation process brings a real improvement.

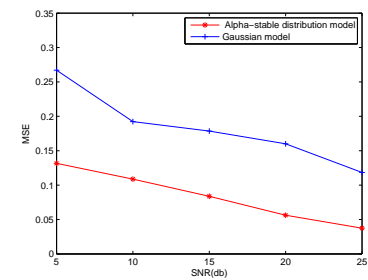

Fig. 3. MSE of the multicarrier signal estimate versus SNR.

Thus, simulations demonstrate the efficiency of the proposed method for the joint estimation of the multicarrier signal and noise parameters.

\section{CONCLUSIONS}

In this paper, we deal with the problem of impulsive interference mitigation in ad hoc networks. In this context, we consider more particularly an OFDM transmission link. In the proposed model, we assumed that the signal distortion due to MAI is represented by a symmetric $\alpha$-stable distribution. We propose an approach based on Bayesian estimation using particle filtering, to jointly estimate the multicarrier signal and the noise parameters. Based on the performed results, it can be firstly concluded that $\alpha$-stable distributions are well suited to model the noise that exhibit impulsive nature. Furthermore, the efficiency and the robustness of the proposed algorithm for interference mitigation have been demonstrated.

\section{REFERENCES}

[1] G. Bresler, A. Parekh, and D.N.C. Tse, "The approximate capacity of the many-to-one and one-to-many gaussian interference channels," IEEE Transactions on Information Theory, vol. 56, no. 9, pp. 4566-4592, September 2010.

[2] R. Dabora, I. Maric, and A. Goldsmith, "Relay strategies for interference-forwarding," in IEEE Information Theory Workshop ITW '08, 2008, pp. 46-50.

[3] E. Sousa, "Performance of a spread spectrum packet radio network in a poisson field of interferers," IEEE Transaction on Information Theory, vol. 38, no. 6, pp. 17431754, November 1992.

[4] J. Ilow and D. Hatzinakos, "Impulsive noise modeling with stable distributions fading environments," in 8th IEEE Signal Processing Workshop on Statistical Signal and Array Processing, June 1996, p. 140143.

[5] M.Z. Win, P.C. Pinto, and L.A. Shepp, "A mathematical theory of network interference and its applications," Proceedings of the IEEE, vol. 97, no. 2, pp. 205-230, February 2009.

[6] H. El Ghannudi, L. Clavier, N. Azzaoui, F. Septier, and P.A. Rolland, "Alpha-stable interference modeling and cauchy receiver for an ir-uwb ad hoc network," IEEE Transactions on Communications, vol. 58, no. 6, pp. 1748-1757, June 2010.

[7] C.L. Nikias and M. Shao, Signal Processing with AlphaStable Distributions and Applications, John Wiley and Sons, 1995.

[8] F. Septier, Y. Delignon, A. Menhaj-Rivenq, and C. Garnier, "Nonpilot-Aided Sequential Monte-Carlo Method to Joint Signal, Phase Noise and Frequency Offset Estimation in Multicarrier Systems," Eurasip Journal on Advances in Signal Processing, vol. 2008, 2008.

[9] D.F. Andrews and C.L. Mallows, "Scale mixtures of normal distributions," Journal of the Royal statistical Society B, vol. 36, pp. 99-102, 1974.

[10] M. Lombardi and S.J. Godsill, "On-line bayesian estimation of signals in symmetric alpha-stable noise," IEEE Transactions on Signal Processing, vol. 54, pp. 775-779, February 2006.

[11] A. Doucet, N. De Freitas, and N. Gordon, Sequential Monte Carlo Methods in Practise, Springer-Verlag, 2001. 